\title{
Perceptions on F/OSS Adoption
}

\author{
Bulent Ozel ${ }^{1}$, Uros Jovanovic ${ }^{2,}$, Beyza Oba ${ }^{3}$, and Manon van Leeuwen ${ }^{4}$ \\ 1 Carnegie Mellon University, Pittsburgh, USA, bulento@cmu.edu \\ 2 XLAB, Ljubljana, Slovenia, uros.jovanovic@xlab.si \\ 3 Istanbul Bilgi University, Istanbul, Turkey, boba@bilgi.edu.tr \\ 4 FUNDECYT, Badajoz, Spain, manon@fundecyt.es
}

\begin{abstract}
This paper aims to reveal results of a survey run by the $\operatorname{tOSSad}^{1}$ project. The majority of survey variables devised to capture perception of public administrators around Europe regarding the importance they attach to the factors such as F/OSS product quality, availability of support, expertise and documentation, TCO, vendor lock-in, political influence, administrative attitudes, productivity, and training costs, all of which intermingle with financial, technical, legal, and personal issues. The analysis consist of depiction of respondents' administration profile in terms of their F/OSS usage and adoption, descriptive summary and analyses of factors mentioned above, and statistical inferential analyses of survey items. Some valid statistical tests are conducted to understand, to discuss and to see the extend and significance of any F/OSS adoption generalizations for Europe based on the findings of this particular survey.
\end{abstract}

\section{Introduction}

Survey respondents are selected from public bodies at middle to high level of managerial positions. A prior knowledge or experience on F/OSS of administrators has been an implicit factor in selecting them as respondents for the survey. However, respondents' F/OSS knowledge depth, their experience level and type, software acquisition policy of their administration, their administrative or personal attitude towards F/OSS, etc have been disregarded at the phase of convenience sampling. The survey covers 13 different countries around Europe with a slightly varying number of respondents for each of them. hey are Ukraine, Turkey, Sweden, Spain, Slovenia, Norway, Malta, Italy, Germany, Bulgaria, Austria, France, and Netherlands. The number and regional diversity of our sampling allows us to run some key descriptive and inferential statistical methods.

\section{Analysis}

The analyses we have run consist of depiction of respondents' administration profile in terms of their F/OSS usage and adoption, descriptive summary and analyses of

1 tOSSad is an EU project funded under FP6 IST program, www.tossad.org.

Please use the following format when citing this chapter.

Ozel, B., Jovanovic, U., Oba, B. and van Leeuwen, M., 2007, in IFIP International Federation for Information Processing, Volume 234, Open Source Development, Adoption and Imnovation, eds. J. Feller, Fitzgerald, B., Scacchi, W., Sillitti, A., (Boston: Springer), pp. 319-324 
factors mentioned above, and statistical inferential analyses of survey items. Some valid statistical tests are conducted to understand, to discuss and to see the extent and significance of any F/OSS adoption generalizations for Europe based on the findings of this particular survey. Most of the survey items are five-point Likert scaled $[1,2]$. All figures, statistical summaries and tests are generated or devised manually using $\mathrm{R}$ Project2, a very detailed analysis and discussion of this survey is available at the tOSSad project Web portal.

\subsection{Descriptive Results}

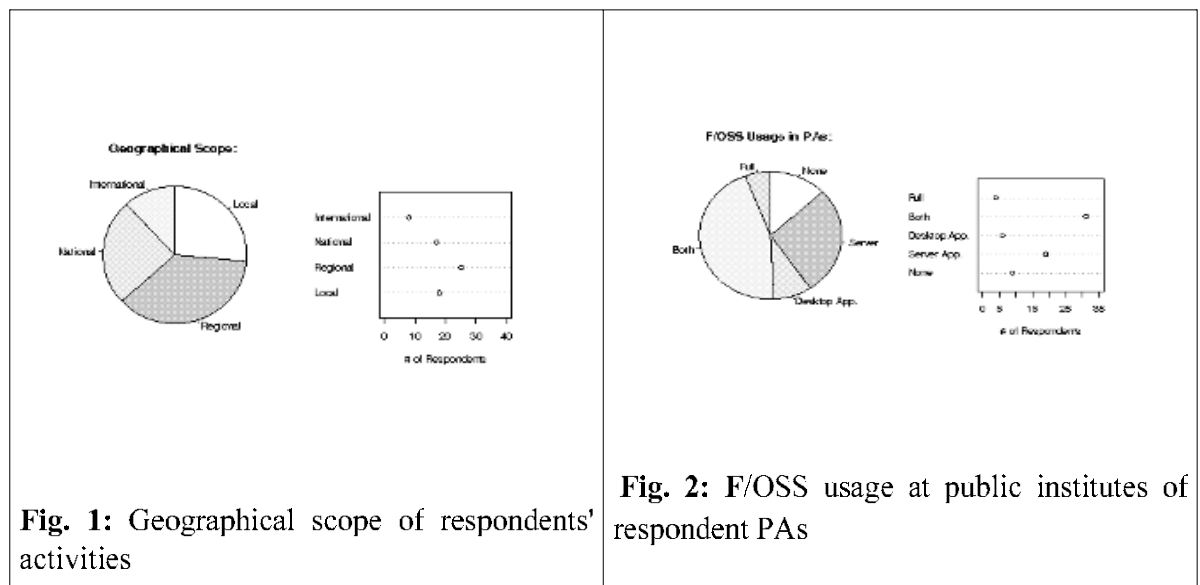

The vast majority of the survey respondents are working in public organizations which have either national or regional level activities. Although a knowledge and acquaintance on F/OSS has been a loose precondition in our sampling, 9 of them, out of 69 valid respondents, declared that they do not use any F/OSS product within their organization or it is not clear to their knowledge. Along with F/OSS usage at any level, we have asked whether our respondents are actively participating, collaborating, or supporting any $\mathrm{F} / \mathrm{OSS}$ related projects. It is seen that about $\% 77$ of the survey respondents have taken part in F/OSS related projects. We have seen that F/OSS project participation of public administrations parallels its adoption within their organization which is an inherently expected picture.

With the first group of survey items we have attempted to understand the importance of quality, support, and documentation in views of the respondents towards F/OSS adoption at their public institutes. A summary of responses are given on Figures 3, 4, and 5. Apparently, we can conclude that F/OSS product quality alone has a direct impact on its adoption. World wide observations elsewhere[3] also support that fact, for instance, increase of F/OSS usage on desktops entangles with its progress in becoming more and more end user friendly. Importance attached to

2 http://www.r-project.org/ 
accessibility of expertise and support, availability of documentation have a rather bell shape distributions which imply that they are not a significant factor as a F/OSS adoption barrier. This picture might be an important input to the debate about documentation and manuals necessary for F/OSS migration in Public Administrations. Considering the fact that respondents of this survey have an experience or knowledge on F/OSS and an the fact that a major group of them have deployed or have been involved in F/OSS deployment within their administration we can say that our study rather supports the view that availability of books, manuals and other documentation is not an important barrier factor against F/OSS adoption in public bodies.

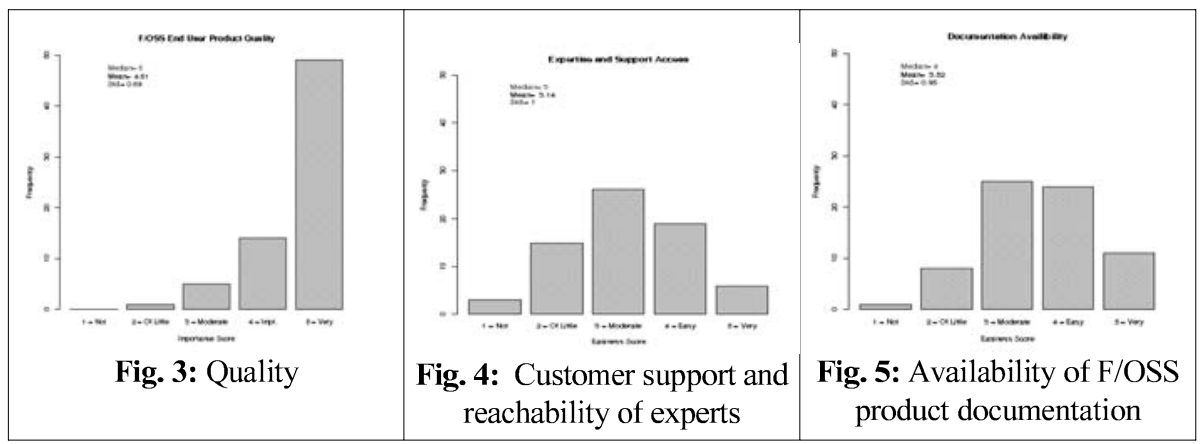

A group of survey items have been placed within the survey to examine effect of built-in barriers such as political decisions, administrative and personal attitudes towards F/OSS in general. This finding shows that along with technological, service, and acquirement costs 'political influence' is also a determinant factor in F/OSS deployment within public administrations. Furthermore, it is interesting to notice that even though responses to other technological and financial factors we have examined so far show a moderate impact on F/OSS adoption, political influence as a factor in the issue has a relatively sharper implication of being a barrier.

Respondents are asked to rate first their attitudes then attitude of their administration about F/OSS. We have seen that the positive personal attitude of the respondents migrate to relatively more negative levels when they are asked to rate general attitude of their administration about F/OSS. We can interpret about this discrepancy in several ways. The first explanation might drive upon the fact that F/OSS advocacy or acceptance is rather more common at personal level than at institutional level. In fact, diffusion of innovations which is theorized and formalized by Everett Rogers[4] supports the observation of our survey which is in line with that fact. When we assume that F/OSS as a whole is an innovative technology, then according to diffusion of innovations theory educated individuals with multiple info sources have greater propensity to take risk in evaluating, trying, and adopting new technologies. A second explanation of such a discrepancy might partially stem from the unavoidable bias that might occur in a survey study [2]. It is highly probable that we have been responded by individuals with relatively more affinities to F/OSS, that 
is, by those having higher willingness and ability to adopt F/OSS depending on their awareness and interest.

The respondents are asked whether they have faced any problem while introducing F/OSS at their administration. Those with negative answers are further asked to explain the reason(s) of their bad experience(s). Textual analysis of their answers reveals four basic sources of bad experience at the introduction phase of F/OSS deployment in public administrations. The first one, the most common as well, is the resistance to change. The second one is the lack of visions on F/OSS business model. Third one is its unsatisfactory compatibility with proprietary file formats. The last reported source of bad experience is lack of satisfactory GUI desktop distributions.

\subsection{Inferential Analysis}

Having processed responses to the survey in the form of raw frequency counts, we have applied Chi-Square nonparametric significance tests to see whether or not there are any significant relationships in between having signed a F/OSS provision contract and F/OSS adoption factors/issues such as overall F/OSS introduction experience, political influence, administrative attitude, employee training, affordability and TCO. We have observed that public officers who involve in legal F/OSS provision process face significantly more negative attitudes from their administration. The summary of that test is given in Table 1 .

Table 1: F/OSS provision contracts and administrative attitude

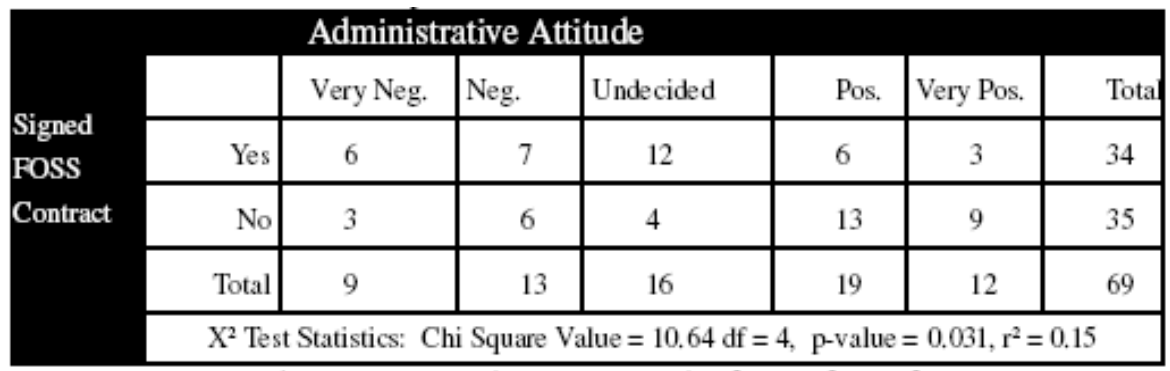

We have combined survey items on access to expertise, support and documentation to create a single averaged 'accessibility' latent item. Figure 6 (a) shows that new combined response displays a normal like distribution with slightly positive kurtosis $($ std $=0.81)$ around the mean $\left(L_{-}=3.33\right)$. Then, we have grouped averaged responses to determine any opinion and experience differences in between group of respondents who have signed a F/OSS provision contract and those who have not. The normality check for each test group is performed using the normal QQ plots. Next, we have examined whether we can infer any significant relation in between the experience or opinion about availability of expertise, support, documentation on F/OSS and having signed a contract on any F/OSS deployment at 
public administrations. We have used a two-sided Welch Two Sample T-test[5]. We have observed a significant correlation $(p$-value $=0.00026)$. In order to examine the direction of relation we have observed response distribution of each group. It shows that those who have signed F/OSS in order to install or acquire additional F/OSS products acknowledge the easy access to expertise, support, and documentation which is necessary for organizational level F/OSS adoption.

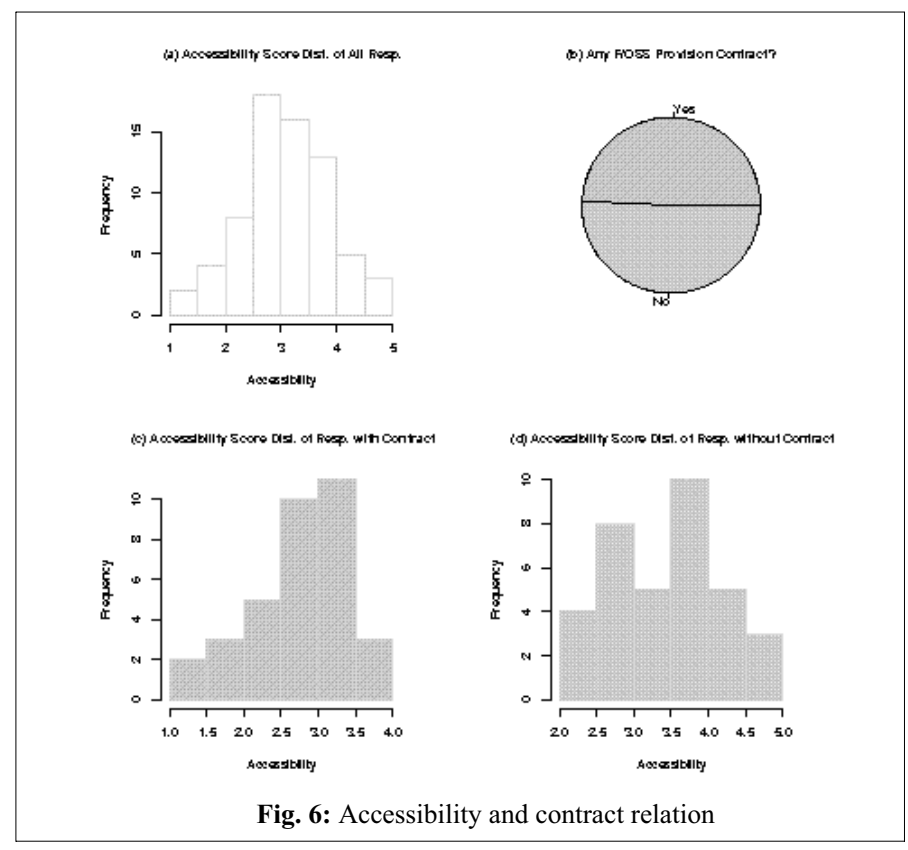

Following the same pattern given above we have generated another latent item 'attitude' which combines items on personal attitude and administrative attitude about F/OSS. Then, again, we have split the respondents according whether they have signed F/OSS provision contract or they have not. The t-test suggests that there is a statistically significant relationship ( $p$-value $=0.006$ ) in between developing general attitudes about F/OSS adoption and the experience of having signed F/OSS provision at public organizations. Our further analysis shows that formed attitudes are based on the particular experience of respondents. Those who have signed a contract while introducing F/OSS at their administration has apparently declare existence of more negative attitudes about F/OSS.

We finally have examined whether there is a correlation in between general F/OSS attitudes and availability of F/OSS expertise, service, and documentation. We have run nonparametric Spearman's rank correlation test[6, 7]. Our test specifically checks the availability of a positive correlation in between having easy access to F/OSS expertise, customer service, and documentation and developing a positive attitude towards F/OSS introduction. Expectedly, an estimated $p=0.003$ " $=0.05$ indicates that there is a statistically significant positive relation but the correlation is 
far from perfect. This relatively low correlation rho $=0.32$ implies that administrative F/OSS attitude is not solely based on the service and it suggests that other factors such as political influence, resistance to change, and initial financial barriers should be considered.

\section{Conclusions}

The conclusions we derive hereby are tentative answers to those particular relationships we have investigated but it indicates that the relationships found in our survey can be expected to exist in at larger level in Europe. Yet this particular study reveals the observation that decision takers' and practitioners' perceptions about F/OSS alone do matter towards a larger F/OSS adoption at Public Organizations. However, this study urges the necessity of a larger geographical scope and higher survey responses in number with evenly distributed samplings to derive more statistically confident observations. We hope that this study will lead further detailed and specific surveys about perceptional issues at F/OSS technology adoption.

\section{References}

[1]Likert, R. (1932). "A Technique for the Measurement of Attitudes" Archives of Psychology 140, 55.

[2] Ross, K. C. (1996). Air University Sampling and Surveying Handbook. University Press of the Pacific.

[3] Ghosh, R. et al. (2002) "Free/Libre and Open Source Software: Survey and Study. Part 2B: Open Source Software in the Public Sector"

[4] Rogers, E. M. (2003). Diffusion of Innovation, Fifth Edition. New York, NY: Free Press.

[5] Welch, B. L. (1947). The generalization of "student's" problem when several different population variances are involved. Biometrika. Vol. 34, pp. 28-35.

[6] Best, D. J. and Roberts, D. E. (1975). Algorithm AS 89: The Upper Tail Probabilities of Spearman's rho. Applied Statistics, 24, 377-379.

[7] Hollander, M. and Wolfe, D. A. (1973). Nonparametric statistical inference. New York: John Wiley \& Sons. Pages 185-194. 